\title{
Conic Projections with Three or More Standard Parallels
}

\author{
Miljenko Lapaine \\ University of Zagreb, Faculty of Geodesy - mlapaine@geof.hr
}

\begin{abstract}
The basic property of all map projections is the distribution of inevitable distortions. Conic projections with one or two standard parallels are mentioned in the literature. These are parallels with the property that the distortion of length, area and angles equals zero at each of their points. It turns out that there are conic projections with no standard parallels, as well as those with more than two standard parallels. Such projections exist not only in theory, but examples of such projections can also be constructed.
\end{abstract}

Keywords: map projection, conic projection, standard parallel

\section{Introduction}

The term "conic projection" refers to any projection in which meridians are mapped to equally spaced lines radiating out from the apex and circles of latitude (parallels) are mapped to circular arcs centred on the apex (Wikipedia 2021). When making a conic map, the map maker arbitrarily picks two standard parallels. Those standard parallels may be visualized as secant lines where the cone intersects the globe - or, if the map maker chooses the same parallel twice, as the tangent line where the cone is tangent to the globe. The resulting conic map has low distortion in scale, shape, and area near those standard parallels. Distances along the parallels to the north of both standard parallels and to the south of both standard parallels are stretched; distances along parallels between the standard parallels are compressed. When a single standard parallel is used, distances along all other parallels are stretched (Wikipedia 2021).

Two statements in the previous sentences are invalid. One is that standard parallels can be visualized as crosssectional parallels in which the cone intersects the globe. It has been explained several times that this is not the case (Lapaine 2017, Lapaine and Menezes 2020). Another claim is that in conic projections there are (at most) two standard parallels. This is not true either since conic projections can have more than two standard parallels. This will be discussed in more detail in the paper.

Let us recall that a few years ago a paper was published on azimuthal projections with several standard parallels (Lapaine 2015). According to this work, "This paper shows that relating the projection plane to a projecting sphere does not make much sense. In fact, it can be demonstrated that an azimuthal projection with two, three and more standard parallels exists. How does one explain a plane intersecting a sphere in three concentric circles? Obviously, this is not possible. Of course, such an azimuthal projection is unlikely to be applied widely. It was developed only to show how awkward and unnecessary it is to relate the projection plane to the sphere so that projection distortions can be explained. Furthermore, conic projections with any number of standard parallels can be created in the same way."
Since no one else has so far investigated conic projections with several standard parallels, we will do so in this paper. To our knowledge, only Kavrayskiy (Каврайский 1959) discussed the distribution of distortions in conic projections in general, i.e. not in special cases such as conformal, equivalent or equidistant projections. Unfortunately, he was incorrect. According to him, the graph showing the local linear scale factor along the parallels in conic projections as a function of latitude looks like the one in Figure 1. In this paper, we show that this is not always the case. Kavrayskiy uses the notation $n$ for the local factor of the linear scale along the parallels, while in this paper the notation is $k$, and $n$ is the notation for the factor along the longitude $\lambda$, see equations (1).

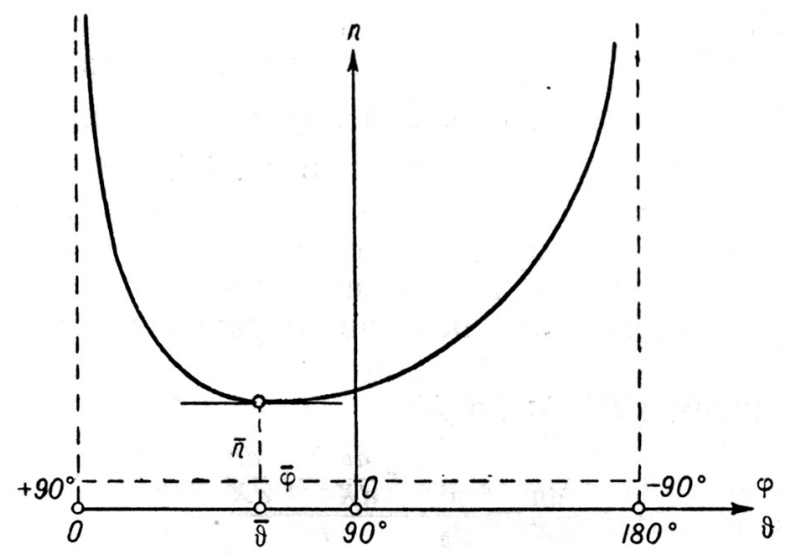

Figure 1. Graph showing the size of the local linear scale factor along the parallels in conic projections according to Kavrayskiy (Каврайский 1959, p. 12). In this article, we show that this is not always the case.

Normal aspect conic projections with one or two standard parallels are usually mentioned in the literature. This paper shows that there are normal aspect conic projections without standard parallels, as well as with three or more standard parallels.

In the second section, we remind you that in addition to the main scale, there is a local linear scale, which at each point depends on the direction. It is a key term by which we define locations with a distortion equal to zero, and 
standard lines in particular. In the third section we deal with conic projections and give formulas by which we investigate standard parallels for such projections. An example is given of a conic projection equidistant along meridians that has no standard parallels, and then an example of a conic projection equidistant along parallels that has one standard parallel. Conic projections with three and eight standard parallels, respectively, are constructed in two sections that follow. The paper ends with the conclusion.

\section{Map scale}

The map scale is not the ratio of the distance on the map to the corresponding distance on the ground, as we can find in many textbooks, but the ratio of the distance on the map to the corresponding distance on the sphere or ellipsoid. Such scale is known as the principal (linear) scale. The principal scale is usually indicated on maps because it determines the general degree of reduction of the length on the map. On most maps, it is usually simply called the 'scale' and is known as the map scale.

The map projections theory says that the map scale changes from point to point, and usually depends on direction. This is the local scale. The local linear scale factor $c(\alpha)$ is the ratio of the differential of the curve arc in the plane of projection and the differential of the corresponding curve arc on the ellipsoid or spherical surface in a given direction $\alpha$.

To avoid misunderstanding, we will define a standard point as the point with

$$
c(\alpha)=1, \text { for all } \alpha \in[0,2 \pi] .
$$

Such a point is usually known as a zero-distortion point. Furthermore, if at all points of a curve we have (1), then it is a zero-distortion curve, or a standard curve. For example, it could be a standard parallel, or a standard meridian.

Not only are standard parallels correct in scale along the parallel; they are correct in every direction. Thus, there is no angular distortion, and conformality exists along these standard parallels, even on an equal-area projection (Snyder 1987).

If we are aware that the representation of the local linear scale factor $c(\alpha)$ is an ellipse, known as a Tissot indicatrix, then the relation (1) says that the ellipse of distortion is a unit circle. Let us emphasize that "for each $\alpha \in[0,2 \pi]$ " in (1) is important. In other words, in order for an ellipse to be a circle, it is necessary for all of its radiuses to be mutually equal or, in the same way, for the semi-axes to be of the same length. It is not enough for only one semi-axis of the ellipse to be equal 1 for the ellipse to be a unit circle.

The requirement (1) is obviously equivalent to the condition

$$
c_{\min }=c_{\max }=1 .
$$

In a special case, when images of meridians and parallels intersect at right angles, the expression (2) can be written like this

$$
h=k=1 \text {, }
$$

where $h$ and $k$ are local linear scale factors along the meridian, and along the parallel, respectively.

\section{Conic projections}

Normal aspect conic projections are those projections in which the images of the parallels are concentric arcs of circles, and the images of the meridians are straight lines passing through the centre of the images of the parallels. The equations in the polar system of the normal aspect conic projection of a sphere of radius $R$ are as follows

$$
\rho=\rho(\varphi), \delta=n \lambda,
$$

where $\varphi \in\left[-\frac{\pi}{2}, \frac{\pi}{2}\right]$ is latitude and $\lambda \in[-\pi, \pi]$ longitude, $n \in(0,1)$. The function $\rho=\rho(\varphi)$ should be continuous, derivable and monotone.

The local linear scale factor along a meridian is

$$
h(\varphi)=-\frac{d \rho}{R d \varphi} .
$$

The local linear scale factor along a parallel is

$$
k(\varphi)=\frac{n \rho}{R \cos \varphi} .
$$

We see that the local linear factors along meridians and parallels are functions only of the latitude. If for some point with coordinates $\varphi, \lambda$ holds

$$
h(\varphi)=k(\varphi)=1,
$$

then it is a standard point. However, since relation (7) if valid holds for every $\lambda$, this $\varphi$ satisfying (7) will be the latitude of the standard parallel.

Here are a few simple conclusions about standard parallels of normal aspect conic projections:

- For conformal normal aspect conic projections $(h(\varphi)=k(\varphi))$, the standard parallel will be determined by $h(\varphi)=1$ or $k(\varphi)=1$.

- For equal-area normal aspect conic projections $(h(\varphi) k(\varphi)=1)$, the standard parallel will be determined by $h(\varphi)=1$ or $k(\varphi)=1$.

- For normal aspect conic projections equidistant along parallels $(k(\varphi)=1)$, the standard parallel will be determined by $h(\varphi)=1$.

- For normal aspect conic projections equidistant along meridians $(h(\varphi)=1)$, the standard parallel will be determined by $k(\varphi)=1$.

- We cannot make any conclusions about the number of standard parallels in normal aspect conic projections, as we could with cylindrical ones (Lapaine, Menezes 2020). This is because for a given $n$ there are infinitely many continuous functions $\rho=\rho(\varphi)$, which are differentiable with positive values and monotone decreasing for $\varphi \in\left[0, \frac{\pi}{2}\right]$, and which satisfy conditions (7) for several different values of the latitudes $\varphi \in\left[0, \frac{\pi}{2}\right]$.

Note that the denominator in the expression (6) has the function cosine. The zero-division problem for $\varphi= \pm \frac{\pi}{2}$ will arise. This is an irregular point of geographical parameterization of the sphere, so we will exclude it from further derivations concerning distortions. 
In the following examples, we will select $\varphi \in\left[0, \frac{\pi}{2}\right), \lambda \in$ $[-\pi, \pi], R=1$ and $n=\frac{1}{2}$.

For $h(\varphi)=1$ it must be

$$
\rho^{\prime}(\varphi)=\frac{d \rho}{d \varphi}=-1,
$$

while for $k(\varphi)=1$ it must be

$$
\rho(\varphi)=2 \cos \varphi \text {. }
$$

\section{Conic projection equidistant along meridians}

Let us consider the normal aspect conic projection given by $\rho=\frac{\pi}{2}-\varphi, \delta=\frac{1}{2} \lambda$.

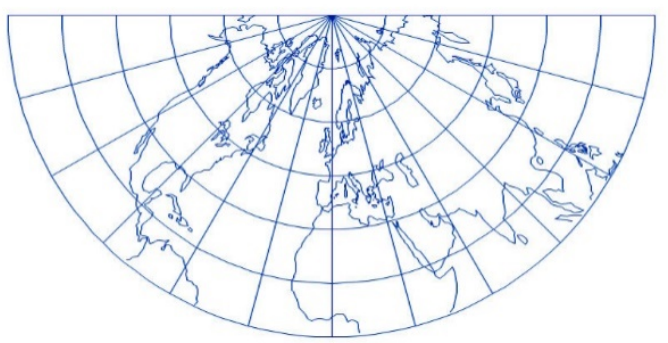

Figure 2. Conic projection equidistant along meridians,

$$
\rho=\frac{\pi}{2}-\varphi, \delta=\frac{1}{2} \lambda
$$

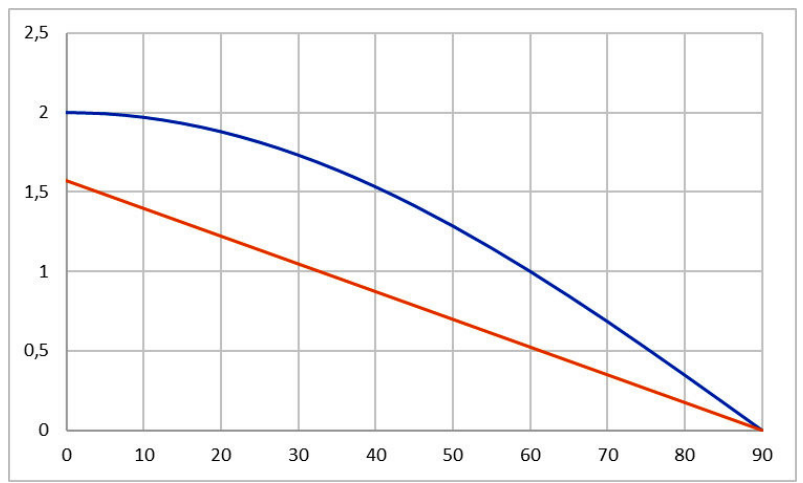

Figure 3. Graph of the function $\rho=2 \cos \varphi$ (blue) and conic projection equidistant along meridians $\rho=\frac{\pi}{2}-\varphi$ (red)

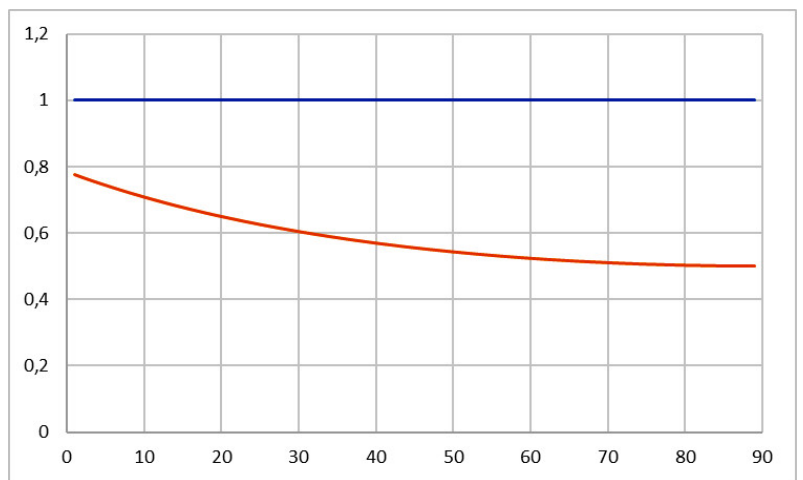

Figure 4. $h=-\frac{d \rho}{d \varphi}=1$ (blue), $k=\frac{n \rho}{\cos \varphi}=\frac{\frac{\pi}{2}-\varphi}{2 \cos \varphi}$ (red), there are no standard parallels for the selected conic projection equidistant along meridians
This mapping is equidistant along the meridians because it is obviously $h(\varphi)=-\frac{d \rho}{d \varphi}=1$. The northern hemisphere of the Earth in this projection is shown in Figure 2. The graph of the function $\rho=2 \cos \varphi$ (blue) and the conic projection equidistant along meridians $\rho=\frac{\pi}{2}-\varphi$ (red) are shown in Figure 3. Figure 4 shows the functions $h=$ $-\frac{d \rho}{d \varphi}=1$ (blue) and $k=\frac{n \rho}{\cos \varphi}=\frac{\frac{\pi}{2}-\varphi}{2 \cos \varphi}$ (red). These two functions have no points in common, from which we conclude that the considered conic projection equidistant along meridians has no standard parallels. For this projection, each meridian is equidistantly mapped, and no parallel is standard (Figure 4).

\section{Conic projection equidistant along parallels}

Let us consider the normal aspect conic projection given by $\rho=2 \cos \varphi, \delta=\frac{1}{2} \lambda$. This mapping is equidistant along parallels because it is obviously $k(\varphi)=1$. The northern hemisphere of the Earth in this projection is shown in Figure 5. The graph of the function $\rho=2 \cos \varphi$ is identical to the graph of the conic projection equidistant along parallels and is represented in Figure 6. Figure 7 shows the functions $h=-\frac{d \rho}{d \varphi}=2 \sin \varphi$ (blue) and $k=\frac{n \rho}{\cos \varphi}=1$ (red). These two functions intersect at one point corresponding to $\varphi=30^{\circ}$. Thus, for this projection, each parallel is mapped equidistantly, and one of these parallels is also a standard one (Figure 7).

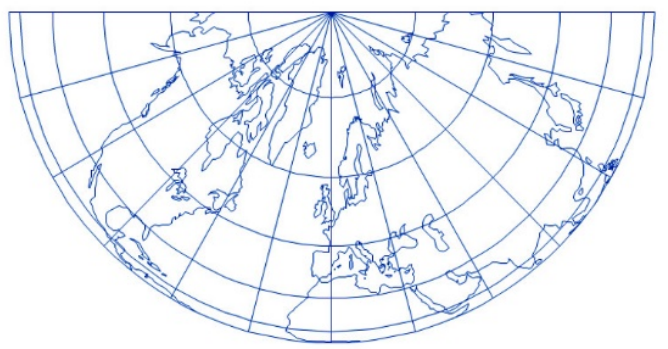

Figure 5. Conic projection equidistant along parallels, $\rho=2 \cos \varphi, \delta=\frac{1}{2} \lambda$

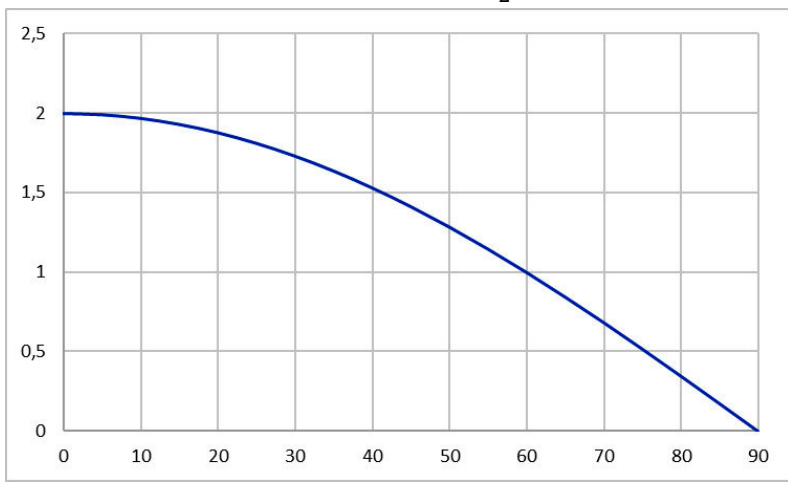

Figure 6. Graph of the function $\rho=2 \cos \varphi$ of the conic projection equidistant along parallels 


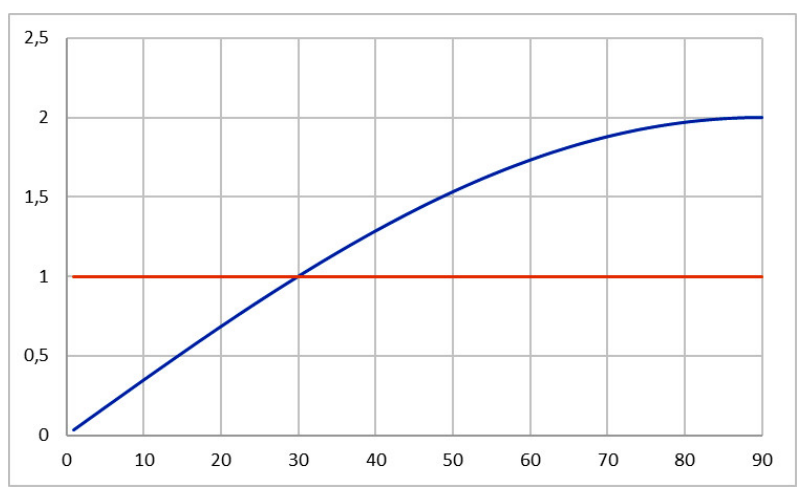

Figure 7. $h=-\frac{d \rho}{d \varphi}=2 \sin \varphi$ (blue), $k=\frac{\rho}{2 \cos \varphi}=1$ (red)

\section{Conic projection with 3 standard parallels}

Let us take three parallels with latitudes $\varphi_{1}, \varphi_{2}$ and $\varphi_{3}$ $\left(0 \leq \varphi_{1}<\varphi_{2}<\varphi_{3} \leq \frac{\pi}{2}\right)$ and $n(0<n<1)$. We are looking for a conic projection for which these three parallels will be standard. The following should be valid for this function:

$$
\begin{aligned}
& h\left(\varphi_{1}\right)=h\left(\varphi_{2}\right)=h\left(\varphi_{3}\right)=1 \text { and } \\
& k\left(\varphi_{1}\right)=k\left(\varphi_{2}\right)=k\left(\varphi_{3}\right)=1 .
\end{aligned}
$$

Thus, the function $\rho=\rho(\varphi):\left[0, \frac{\pi}{2}\right) \rightarrow R$ is required for which (10) holds. Let us try to find a solution with function $\rho=\rho(\varphi)$ consisting of two polynomials of third degree which are connected in $\varphi_{2}$ :

$$
\begin{aligned}
& \rho(\varphi)=a_{1} \varphi^{3}+b_{1} \varphi^{2}+c_{1} \varphi+d_{1} \text { for } 0 \leq \varphi \leq \varphi_{2} \\
& \rho(\varphi)=a_{2} \varphi^{3}+b_{2} \varphi^{2}+c_{2} \varphi+d_{2} \text { for } \varphi_{2} \leq \varphi<\frac{\pi}{2}
\end{aligned}
$$

Note that $h\left(\varphi_{2}\right)$ and $k\left(\varphi_{2}\right)$ can be written in two ways because $\varphi_{2}$ is in the definition area of both polynomials (11) and (12).

We have a total of eight linear equations

$$
\begin{gathered}
n\left(a_{1} \varphi_{1}^{3}+b_{1} \varphi_{1}^{2}+c_{1} \varphi_{1}+d_{1}\right)=\cos \varphi_{1} \\
n\left(a_{1} \varphi_{2}^{3}+b_{1} \varphi_{2}^{2}+c_{1} \varphi_{2}+d_{1}\right)=\cos \varphi_{2} \\
3 a_{1} \varphi_{1}^{2}+2 b_{1} \varphi_{1}+c_{1}=-1 \\
3 a_{1} \varphi_{2}^{2}+2 b_{1} \varphi_{2}+c_{1}=-1 \\
n\left(a_{2} \varphi_{2}^{3}+b_{2} \varphi_{2}^{2}+c_{2} \varphi_{2}+d_{2}\right)=\cos \varphi_{2} \\
n\left(a_{2} \varphi_{3}^{3}+b_{2} \varphi_{3}^{2}+c_{2} \varphi_{3}+d_{2}\right)=\cos \varphi_{3} \\
3 a_{2} \varphi_{3}^{2}+2 b_{2} \varphi_{3}+c_{2}=-1 \\
3 a_{2} \varphi_{2}^{2}+2 b_{2} \varphi_{2}+c_{2}=-1
\end{gathered}
$$

with eight unknown parameters $a_{1}, b_{1}, c_{1}, d_{1}$ and $a_{2}, b_{2}$, $c_{2}, d_{2}$. The first four equations in the system (13) contain the unknowns $a_{1}, b_{1}, c_{1}, d_{1}$, and the other four $a_{2}, b_{2}, c_{2}, d_{2}$. So, we are talking about two systems of four linear equations with four unknowns each. The solutions of these two systems can be written in explicit form

$$
\begin{gathered}
a_{1}=\frac{2}{\left(\varphi_{1}-\varphi_{2}\right)^{2}}\left[\frac{\cos \varphi_{2}-\cos \varphi_{1}}{n\left(\varphi_{1}-\varphi_{2}\right)}-1\right] \\
b_{1}=-\frac{3}{2} a_{1}\left(\varphi_{1}+\varphi_{2}\right) \\
c_{1}=3 a_{1} \varphi_{1} \varphi_{2}-1 \\
d_{1}=\frac{\cos \varphi_{2}}{n}-\left(a_{1} \varphi_{2}^{3}+b_{1} \varphi_{2}^{2}+c_{1} \varphi_{2}\right)
\end{gathered}
$$

$$
\begin{gathered}
a_{2}=\frac{2}{\left(\varphi_{2}-\varphi_{3}\right)^{2}}\left[\frac{\cos \varphi_{3}-\cos \varphi_{2}}{n\left(\varphi_{2}-\varphi_{3}\right)}-1\right] \\
b_{2}=-\frac{3}{2} a_{2}\left(\varphi_{2}+\varphi_{3}\right) \\
c_{2}=3 a_{2} \varphi_{2} \varphi_{3}-1 \\
d_{2}=\frac{\cos \varphi_{3}}{n}-\left(a_{2} \varphi_{3}^{3}+b_{2} \varphi_{3}^{2}+c_{2} \varphi_{3}\right)
\end{gathered}
$$

If we substitute the calculated values of the parameters in (11) and (12) we may have obtained the required solution. Namely, let us notice that for any choice $\varphi_{1}, \varphi_{2}$ and $\varphi_{3}$ $\left(0 \leq \varphi_{1}<\varphi_{2}<\varphi_{3}<\frac{\pi}{2}\right)$ and $n(0<n<1)$ we will not always get the correct map projection in the described way because we did not ensure the condition of monotonicity of the function $\rho=\rho(\varphi)$.

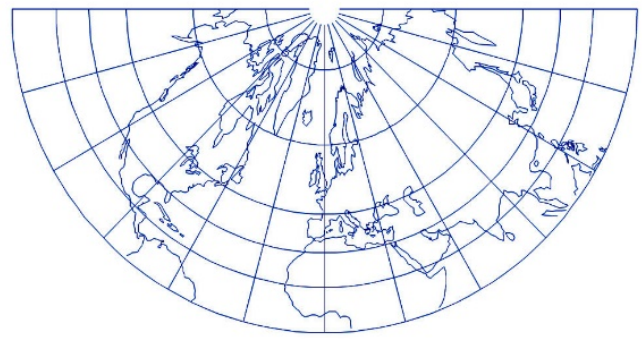

Figure 8. Conic projection with three standard parallels $\left(15^{\circ}\right.$, $40^{\circ}, 85^{\circ}$ ). The parallels are shown with a step of $15^{\circ}$, and the meridians with a step of $30^{\circ}$

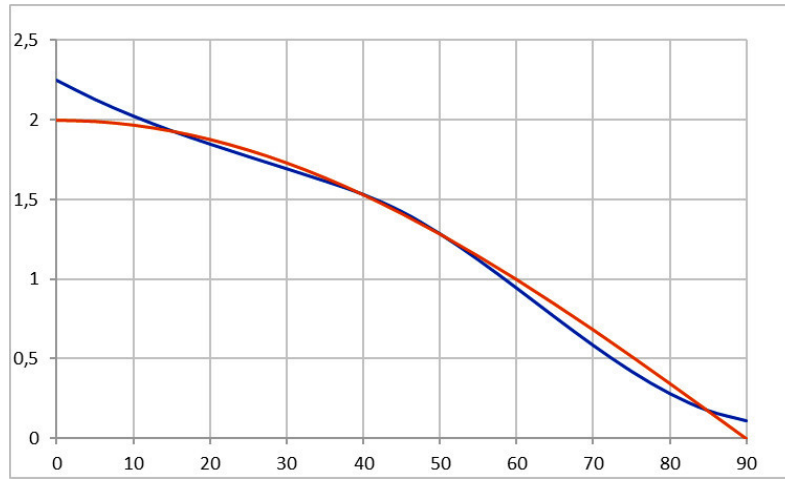

Figure 9. Graph of the function $\rho=2 \cos \varphi$ (red) and function $\rho=\rho(\varphi)$ of the conic projection with three standard parallels $\left(15^{\circ}, 40^{\circ}, 85^{\circ}\right.$, blue $)$

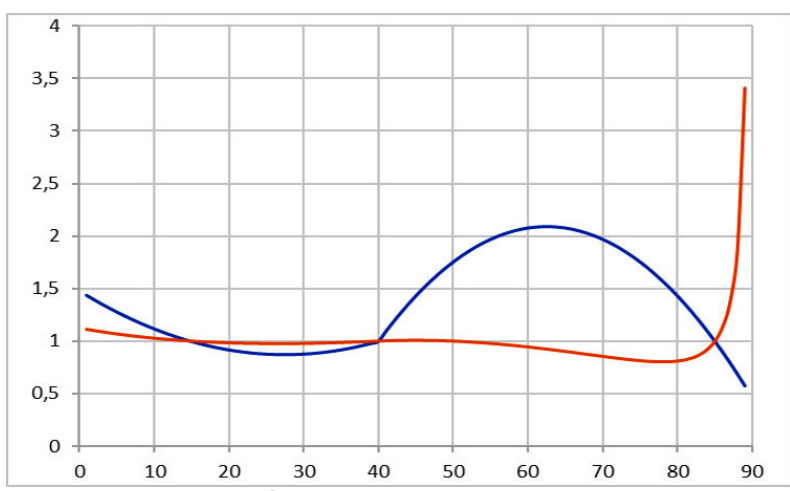

Figure 10. $h=-\frac{d \rho}{d \varphi}$ (blue), $k=\frac{\rho}{2 \cos \varphi}$ (red), for the conic projection with three standard parallels $\left(15^{\circ}, 40^{\circ}, 85^{\circ}\right)$ 
But for some choices of given values, we will get the appropriate solution. For example, if we take $\varphi_{1}=15^{\circ}$, $\varphi_{2}=40^{\circ}, \varphi_{3}=85^{\circ}$ and $n=0.5$ we will get a conic projection with these three standard parallels (see Figures 8-10).

\section{Conic projection with several standard parallels}

Let us take two parallels with latitudes $\varphi_{1}$ and $\varphi_{2}(0 \leq$ $\left.\varphi_{1}<\varphi_{2} \leq \frac{\pi}{2}\right)$ and $n(0<n<1)$. We are looking for a conic projection for which these two parallels will be standard parallels. Let us note

$$
\bar{\varphi}=\frac{\varphi_{1}+\varphi_{2}}{2}
$$

and try to find a solution with function $\rho=\rho(\varphi)$ which is a third-degree polynomial of the form

$$
\rho=a(\varphi-c)^{3}+b(\varphi-c)^{2}+d \text { for } \varphi_{1} \leq \varphi \leq \bar{\varphi} .
$$

Let us determine

$$
\frac{d \rho}{d \varphi}=\rho^{\prime}=3 a(\varphi-c)^{2}+2 b(\varphi-c)
$$

and set these conditions

$$
\begin{aligned}
& n\left[a\left(\varphi_{1}-c\right)^{3}+b\left(\varphi_{1}-c\right)^{2}+d\right]=\cos \varphi_{1} \\
& n\left[a(\bar{\varphi}-c)^{3}+b(\bar{\varphi}-c)^{2}+d\right]=\cos \bar{\varphi} \\
& 3 a\left(\varphi_{1}-c\right)^{2}+2 b\left(\varphi_{1}-c\right)=-1 \\
& 3 a(\bar{\varphi}-c)^{2}+2 b(\bar{\varphi}-c)=0 .
\end{aligned}
$$

Conditions (18) and (20) say that $\varphi_{1}$ will be the latitude of the standard parallel. Condition (19) means that the local linear scale factor in the direction of the parallel for $\bar{\varphi}$ will be equal to $1, k(\bar{\varphi})=1$, and condition (21) that the cubic parabola (16) will have a horizontal tangent (local extreme) in $\bar{\varphi}$. The system of linear equations (18)-(21) is not difficult to solve and the solution is

$$
\begin{gathered}
a=\frac{\frac{2}{n}\left(\cos \bar{\varphi}-\cos \varphi_{1}\right)-\left(\varphi_{1}-\bar{\varphi}\right)}{\left(\varphi_{1}-\bar{\varphi}\right)^{3}} \\
b=\frac{\varphi_{1}-\bar{\varphi}-\frac{3}{n}\left(\cos \bar{\varphi}-\cos \varphi_{1}\right)}{\left(\varphi_{1}-\bar{\varphi}\right)^{2}} \\
c=\bar{\varphi} \\
d=\frac{\cos \bar{\varphi}}{n} .
\end{gathered}
$$

Analogously, we look for the function $\rho=\rho(\varphi)$, which is again a polynomial of third degree as in (16), but defined on the second segment

$$
\rho=a(\varphi-c)^{3}+b(\varphi-c)^{2}+d \text { za } \bar{\varphi} \leq \varphi \leq \varphi_{2} .
$$

The parameters $a, b, c$ and $d$ will be determined analogously to the previously described procedure. The solution will be of the form (22), we only need to put $\varphi_{2}$ instead of $\varphi_{1}$. The described procedure for two parallels with latitudes $\varphi_{1}$ and $\varphi_{2}\left(0 \leq \varphi_{1}<\varphi_{2}<\frac{\pi}{2}\right)$ and $n(0<$ $n<1$ ) can now be easily extended to several parallels $\varphi_{1}$, $\varphi_{2}, \varphi_{3}, \varphi_{4}, \ldots\left(0 \leq \varphi_{1}<\varphi_{2}<\varphi_{3}<\varphi_{4} \ldots<\frac{\pi}{2}\right)$. Only the formulas derived for $\varphi_{1}$ and $\varphi_{2}$ should be applied to $\varphi_{2}$ and $\varphi_{3}$, then to $\varphi_{3}$ and $\varphi_{4}$, and so on.

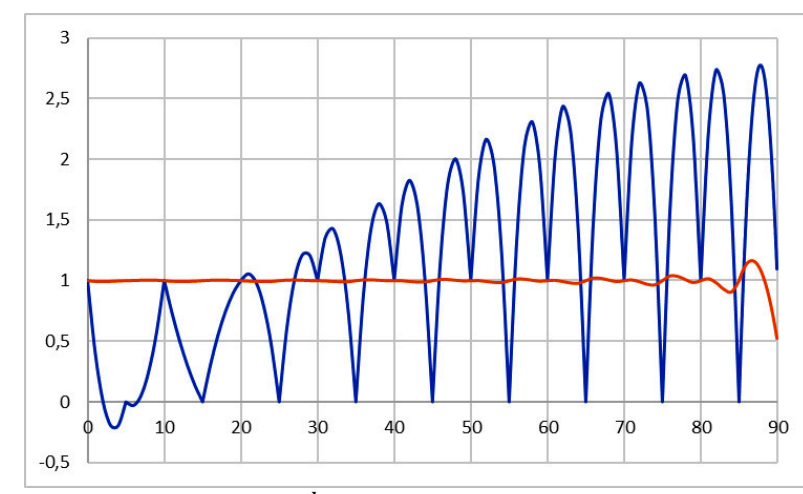

Figure 11. $h=-\frac{d \rho}{d \varphi}$ (blue), $k=\frac{\rho}{2 \cos \varphi}$ (red), for conic projection with 8 standard parallels $\left(10^{\circ}, 20^{\circ}, \ldots, 80^{\circ}\right)$. It holds $\rho(10 i)=2 \cos 10 i, \rho^{\prime}(10 i)=-1, i=1,2, \ldots, 8$. The problem remains on the interval $0^{\circ} \leq \varphi<10^{\circ}$, where a part of the obtained cubic parabola is not monotonically decreasing. Compare Fig. 14.

It is shown that the described procedure can, for example, give a conic projection with 8 standard parallels $\left(10^{\circ}, 20^{\circ}\right.$, $\ldots, 80^{\circ}$ ) for $10^{\circ} \leq \varphi<90^{\circ}$. The problem remains on the interval $0^{\circ} \leq \varphi<10^{\circ}$, where a part of the obtained cubic parabola is not monotonically decreasing (See Figure 11). So, if we are not satisfied with the projection on the interval $10^{\circ} \leq \varphi<90^{\circ}$, but we want instead a projection on the interval $0^{\circ} \leq \varphi<90^{\circ}$, we must extend the equation for $\rho=\rho(\varphi)$ to the interval $0^{\circ} \leq \varphi<10^{\circ}$ in an appropriate way. One possibility is that the function $\rho=$ $\rho(\varphi)$ at the interval $0^{\circ} \leq \varphi<10^{\circ}$ is the upper arc

$$
\rho=c+\frac{b}{a} \sqrt{a^{2}-\varphi^{2}},
$$

of the ellipse

$$
\frac{\varphi^{2}}{a^{2}}+\frac{(\rho-c)^{2}}{b^{2}}=1
$$

where $a, b$, and $c$ are the parameters to be determined. Let us calculate

$$
\rho^{\prime}=\frac{d \rho}{d \varphi}=-\frac{b}{a} \frac{\varphi}{\sqrt{a^{2}-\varphi^{2}}}
$$

and then write

$$
\begin{aligned}
& \rho(0)=c+\frac{b}{a} a=c+b=\frac{1}{n^{\prime}}, \\
& \rho\left(\varphi_{1}\right)=c+\frac{b}{a} \sqrt{a^{2}-\varphi_{1}^{2}}=\frac{\cos \varphi_{1}}{n}, \\
& \rho^{\prime}\left(\varphi_{1}\right)=-\frac{b}{a} \frac{\varphi_{1}}{\sqrt{a^{2}-\varphi_{1}^{2}}}=-1,
\end{aligned}
$$

where we denoted by $\varphi_{1}$ an angle of $10^{\circ}$, or $\frac{\pi}{18}$.

It is not difficult to reduce three equations (27)-(29) with three unknowns $a, b$ and $c$ to one equation with one unknown $a$

$$
\sqrt{a^{2}-\varphi_{1}^{2}}\left(\sqrt{a^{2}-\varphi_{1}^{2}}-a\right)=K
$$

where

$$
K=\frac{\varphi_{1}\left(\cos \varphi_{1}-1\right)}{n} .
$$

From (30) we get

$$
a=\frac{\varphi_{1}^{2}+K}{\sqrt{\varphi_{1}^{2}+2 K}} .
$$


After that, from (27)-(29) we get

$$
b=\frac{a}{\varphi_{1}} \sqrt{a^{2}-\varphi_{1}^{2}} \text { and } c=\frac{1}{n}-b .
$$

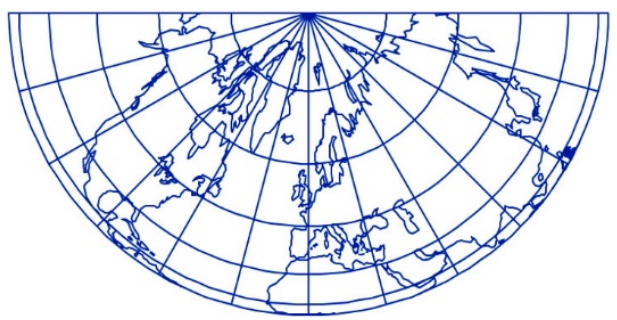

Figure 12. Conic projection with 8 standard parallels $\left(10^{\circ}, 20^{\circ}\right.$, $\left.\ldots, 80^{\circ}\right)$. The parallels are shown with a step of $15^{\circ}$, and the meridians with a step of $30^{\circ}$

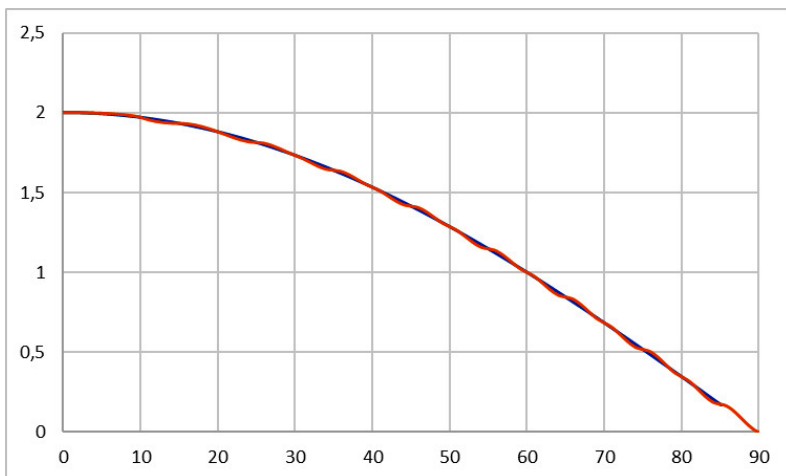

Figure 13. Graph of the function $\rho=2 \cos \varphi$ (blue) and the function $\rho=\rho(\varphi)$ of the conic projection with 8 standard parallels (red)

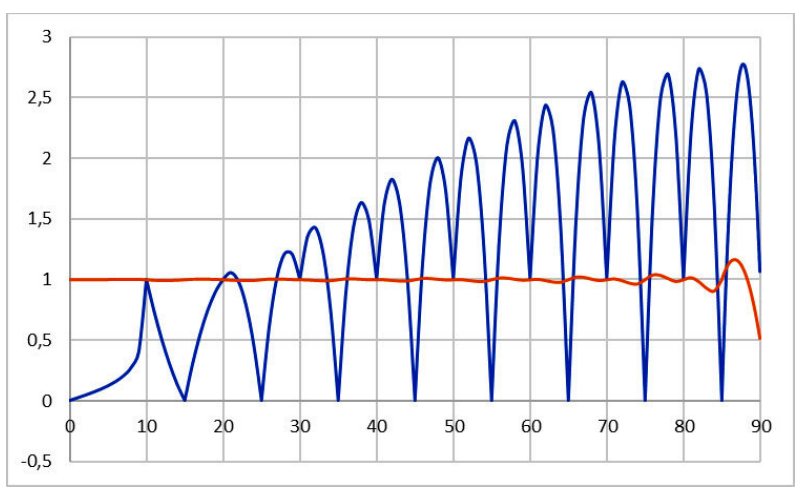

Figure 14. $h=-\frac{d \rho}{d \varphi}$ (blue), $k=\frac{\rho}{2 \cos \varphi}$ (red), for conic projection with 8 standard parallels $\left(10^{\circ}, 20^{\circ}, \ldots, 80^{\circ}\right)$. It holds $\rho(10 i)=2 \cos 10 i, \rho^{\prime}(10 i)=-1, i=1,2, \ldots, 8 . \rho=\rho(\varphi)$ is the arc of the ellipse for latitudes $0^{\circ}$ to $10^{\circ}$, and then cubic parabolas that follow piecewise at every $10^{\circ}$. Compare Fig. 11.

Conic projection with 8 standard parallels $\left(10^{\circ}, 20^{\circ}, \ldots\right.$, $80^{\circ}$ ) and its distortion characteristics are shown in Figures 12-14.

\section{Conclusion}

The basic property of all map projections is the distribution of inevitable distortions. Normal aspect conic projections with one or two standard parallels are mentioned in the literature. These are parallels with the property that at each of their points the distortion of length, area and angle is equal to zero. This paper shows that there are conic projections without a standard parallel, as well as with more than two standard parallels. Such projections not only exist in theory, but examples of such projections are practically constructed in this paper.

Although it could be expected that a larger number of standard parallels gives a better distribution of distortions of a projection, the examples disprove this. Along the standard parallels the distortions are equal to zero, but between them they can become significant. Therefore, the author of this article does not believe that projections with a larger number of standard parallels deserve greater application in practice. However, awareness of the existence of such projections changes the usual approach in teaching and researching map projections. The distribution of distortions does not depend only on the position of standard parallels.

\section{References}

Каврайский В. В. (1959): Избранные труды. - В 2 т. Т. 2., Вып. 2. Математическая картография. Конические и цилиндрические проекции, их применение. изд. Управления начальника Гидрографической службы ВМФ, Москва

Lapaine, M. (2015): Multi Standard-Parallels Azimuthal Projections, in: Cartography - Maps Connecting the World, C. Robbi Sluter, C. B. Madureira Cruz, P. M. Leal de Menezes (Eds.), Springer International Publishing, Series: Publications of the International Cartographic Association (ICA), doi 10.1007/978-3-319-17738-0_3, 33-44

Lapaine, M. (2017): Standard parallels and secant parallels in conic projections, SJCE, Scientific Journal of Civil Engineering, Vol. 6, No. 2, 127-134

Lapaine, M., Menezes, P. (2020): Standard, secant and equidistant parallels / Standardne, presječne i ekvidistantne paralele, Kartografija i geoinformacije, vol 19, br. $34,40-62$

Snyder J P (1987): Map projections: A working manual, Professional Paper 1395, U.S. Government Printing Office

Wikipedia (2021): Map projection / Conic, https://en.wikipedia.org/wiki/Map_projection\#Conic (27 Mar 2021) 